\title{
Impedancia esofágica intraluminal de canal múltiple: una nueva frontera en motilidad
}

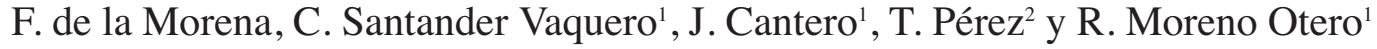 \\ Servicio de Aparato Digestivo. Hospital Infanta Cristina. Parla, Madrid. ${ }^{1}$ Unidad de Motilidad Digestiva. Hospital \\ Universitario de la Princesa y CIBERehd. ${ }^{2}$ Fundación Hospital de Alcorcón. Madrid
}

\section{RESUMEN}

La impedancia esofágica multicanal constituye una de las más recientes herramientas diagnósticas aplicadas a la evaluación de la enfermedad por reflujo gastroesofágico. Esta técnica se basa en la monitorización continua de la conductividad eléctrica en un medio, en este caso el esófago, a través de electrodos situados en la longitud de una sonda, y en los cambios que un elemento como el bolo alimentario puede producir en dicha variable. De esta técnica se derivan nuevas perspectivas en la exploración de la función esofágica y de la enfermedad por reflujo gastroesofágico, hasta ahora sólo cuantificable de forma indirecta por las fluctuaciones generadas por los cambios en el pH del reflujo ácido. Presentamos el caso de una paciente con tos persistente evaluada con este procedimiento, en quien se demostró la presencia de reflujo gastroesofágico débilmente ácido que no pudo ser demostrado previamente con otras exploraciones, y de cómo la impedancia descartó la asociación entre este síntoma y el reflujo no ácido.

Palabras clave: Impedancia esofágica multicanal. Trastorno motor esofágico. Reflujo gastroesofágico.

\begin{abstract}
Multichannel intraluminal impedance is a new technique for evaluating esophageal function and gastroesophageal reflux disease (GERD). This technique relies on the monitoring of electric conductivity in the esophagus though electrodes, and on changes developing in this variable as a result of bolus passage. The proximal distribution and duration of non-acid reflux events can now be quantified, and combined manometry defines the effectiveness of esophageal body function, until now only indirectly evaluated with $\mathrm{pH}$ changes. We discuss the case of a woman with persistent cough in whom intraesophageal multichannel impedance demostrates a minor acid reflux that could not be demonstrated with other diagnostic methods previously, and how this technique excludes any association between cough and non-acid reflux.
\end{abstract}

Key words: Multichannel intraluminal impedance. Esophageal motility disorder. Gastroesophageal reflux.

De la Morena F, Santander Vaquero C, Cantero J, Pérez T, Moreno Otero R. Impedancia esofágica intraluminal de canal múltiple: una nueva frontera en motilidad. Rev Esp Enferm Dig 2008; 100: 86-89.

\section{INTRODUCCIÓN}

La enfermedad por reflujo gastroesofágico (ERGE) es, junto al síndrome de intestino irritable, uno de los grandes retos a los que se enfrenta el especialista de aparato digestivo en su práctica clínica diaria. Esto se debe principalmente a la gran demanda asistencial que su sintoma-

Recibido: 08-10-07.

Aceptado: 29-11-07.

Correspondencia: Felipe de la Morena López. C/ Infanta María, 9, 3 º 7. 28050 Madrid. e-mail: felipe_de_la_morena@hotmail.com tología genera y al elevado coste económico que implica su diagnóstico y tratamiento.

En los últimos años se han diseñado distintas estrategias para un mejor abordaje y control tanto de la sintomatología del reflujo como de las potenciales complicaciones del reflujo ácido. En esta línea, la impedancia intraluminal esofágica de canal múltiple (IIM) ha revolucionado nuestro conocimiento sobre las características del reflujo más allá de las limitaciones del control aislado del $\mathrm{pH}$ mediante $\mathrm{pHme-}$ tría. La IIM permite definir elementos hasta ahora desconocidos como son las características del material refluido y el alcance del reflujo. Además facilita el estudio de factores directamente relacionados con la motilidad como la veloci- 
dad de progresión, el tiempo de aclaramiento y la motilidad sectorial. A este procedimiento se pueden asociar registros convencionales manométricos o, como ocurre en nuestro caso, de $\mathrm{pH}$ consiguiendo informaciones complementarias a las de los registros de impedancia de forma aislada.

A modo de ejemplo exponemos el caso clínico de una paciente con tos crónica, refractaria al tratamiento convencional de la ERGE, estudiada mediante esta técnica de IIM-pH, y como de su resultado se derivan importantes implicaciones en la orientación clínica de la paciente.

\section{OBSERVACIÓN CLÍNICA}

Mujer de 52 años de edad sin antecedentes de interés derivada a nuestra Unidad de Motilidad Digestiva por estudio de tos crónica de 14 años de evolución. La tos presentaba carácter paroxístico, en salvas, no era productiva y tenía predominio en decúbito y con esfuerzos físicos. No asociaba de forma concomitante disfagia ni dolor torácico aunque sí de forma ocasional (1 vez a la semana) pirosis retroesternal con buen control con rabeprazol a dosis de $20 \mathrm{mg}$ cada 24 horas, pero que no modificaba su síntoma principal, la tos.

En el contexto del estudio de la tos la paciente aportaba: Mantoux negativo, radiografía y tomografía axial computerizada de tórax normal, citología y cultivo de esputo negativos, test del sudor en rangos normales y espirometría con prueba broncodilatadora normal. Desde el punto de vista digestivo aportaba una panendoscopia oral y un estudio esófago-gastroduodenal baritado sin anomalías ni evidencia de lesiones por reflujo, así como dos pHmetrías esofágicas de 24 horas realizadas con un año de diferencia. La primera de ellas demostraba la presencia de un reflujo gastroesofágico ácido patológico, mientras que la segunda (efectuada con rabeprazol a dosis de $20 \mathrm{mg}$ cada 12 horas) reveló el adecuado control del reflujo ácido. Ante la sospecha de que la sintomatología estuviera ocasionada por un reflujo de tipo no ácido decidimos realizar una IIM combinada con pHmetría y un estudio manométrico previo.

La manometría esofágica reveló un comportamiento del esfínter esofágico inferior normal con presión de reposo y relajaciones dentro de los límites normales; sin embargo, el cuerpo esofágico en su tercio medio y distal presentaba un $40 \%$ de ondas de amplitud disminuida con el $10 \%$ no transmitidas, lo cual orientaba a nuestra paciente hacia el diagnóstico de un trastorno motor inespecífico del tipo peristalsis esofágica ineficaz en grado moderado.

Los resultados de la IIM-pH bajo su tratamiento actual con rabeprazol $20 \mathrm{mg} /$ día (descendido cuatro meses antes de ser remitida a nuestra unidad por la ausencia de respuesta sintomática demostrada ante dosis más elevadas) demostraron 21 episodios de reflujo ácido distal (detectables con $\mathrm{pH}$ menor de 4; límite hasta 50), 1 episodio de reflujo no ácido (límite hasta 15) y 50 de tipo moderadamente ácido o acid minor (límite de la normalidad 33) con un score de DeMeester de 14,36 (límite patológico 14,72). Sólo 9 de los 50 moderadamente ácidos y 4 de los 21 ácidos alcanzaban el detector (Z1) de impedancia esofágica más proximal, sin que se relacionara ninguno de ellos con un episodio clínico de tos. Como se ha expuesto anteriormente el análisis aislado de los datos de pHmetría realizada en el centro de referencia de la paciente no demostraba reflujo ácido patológico, lo cual fue congruente con los resultados de nuestra IIM-pH, incluso estando la paciente con dosis de rabeprazol menores que en la pHmetría previa. Por otro lado al asociar las determinaciones de $\mathrm{pH}$ al estudio de impedancia se pudo demostrar la presencia muy significativa de reflujos moderadamente ácidos, hecho imposible de detectar con el análisis aislado del registro de $\mathrm{pH}$ (Fig. 1). No obstante ninguno de los episodios de reflujo se asoció a ningún episodio clínico de tos, lo que permitió descartar definitivamente a la ERGE como causa del cuadro clínico, pudiendo de esta manera redirigir las sospechas clínicas hacia otras posibilidades etiológicas de la tos. La paciente se encuentra actualmente en estudio por el servicio de neumología en previsión de un posible origen pleurítico del cuadro tusígeno.

\section{DISCUSIÓN}

La impedancia se define como la propiedad física consistente en la oposición que efectúa un determinado medio al paso de corriente eléctrica a su través. Dicho de otro modo, la impedancia se puede considerar como el opuesto de la conductividad eléctrica. Su valor, cuya unidad es el ohmnio, dependerá de las características fisicoquímicas de la materia (líquido, sólido, gasesoso, ionización) y de la superficie de contacto entre los electrodos y estas sustancias. De todo ello se derivan importantes implicaciones a nivel del conocimiento en modelos biológicos de la motilidad de distintos órganos, en especial de estructura tubular como el aparato digestivo. En ellos la movilidad del bolo alimentario podrá ser estudiada a través de sondas multidetector en distintas posiciones que nos indiquen la progresión del bolo por los cambios en la impedancia entre los distintos sectores. La mayor aportación de esta técnica se deriva de la capacidad no sólo de establecer las características del elemento "móvil" (tipo, velocidad) sino también el sentido de su progresión (anterógrado-retrógrado) a tiempo real, la distancia recorrida y el comportamiento mecánico del tubo digestivo circundante (medidas de aclarado y ausencia de propulsión segmentaria) $(1,2)$.

El registro de impedancia nos ofrecerá un registro temporal continuo de las medidas de resistencia eléctrica del entorno existente entre los detectores situados en la longitud de la sonda. Una vez conocidas las medidas de impedancia de elementos como el aire $(>7.000 \Omega)$, líquidos no iónicos $(<1.500 \Omega)$ e iónicos como el jugo gástrico $(<200 \Omega)$, se obtendrán patrones típicos a través de sonda que demostrarán el paso del bolo alimentario. Así, de forma característica, distinguimos cinco fases (3): la I representa la impedancia basal del esófago sin contenido, la II corresponderá a un ascenso rápido sobre la línea de base que representa la entrada de aire procedente de la deglución, la III 


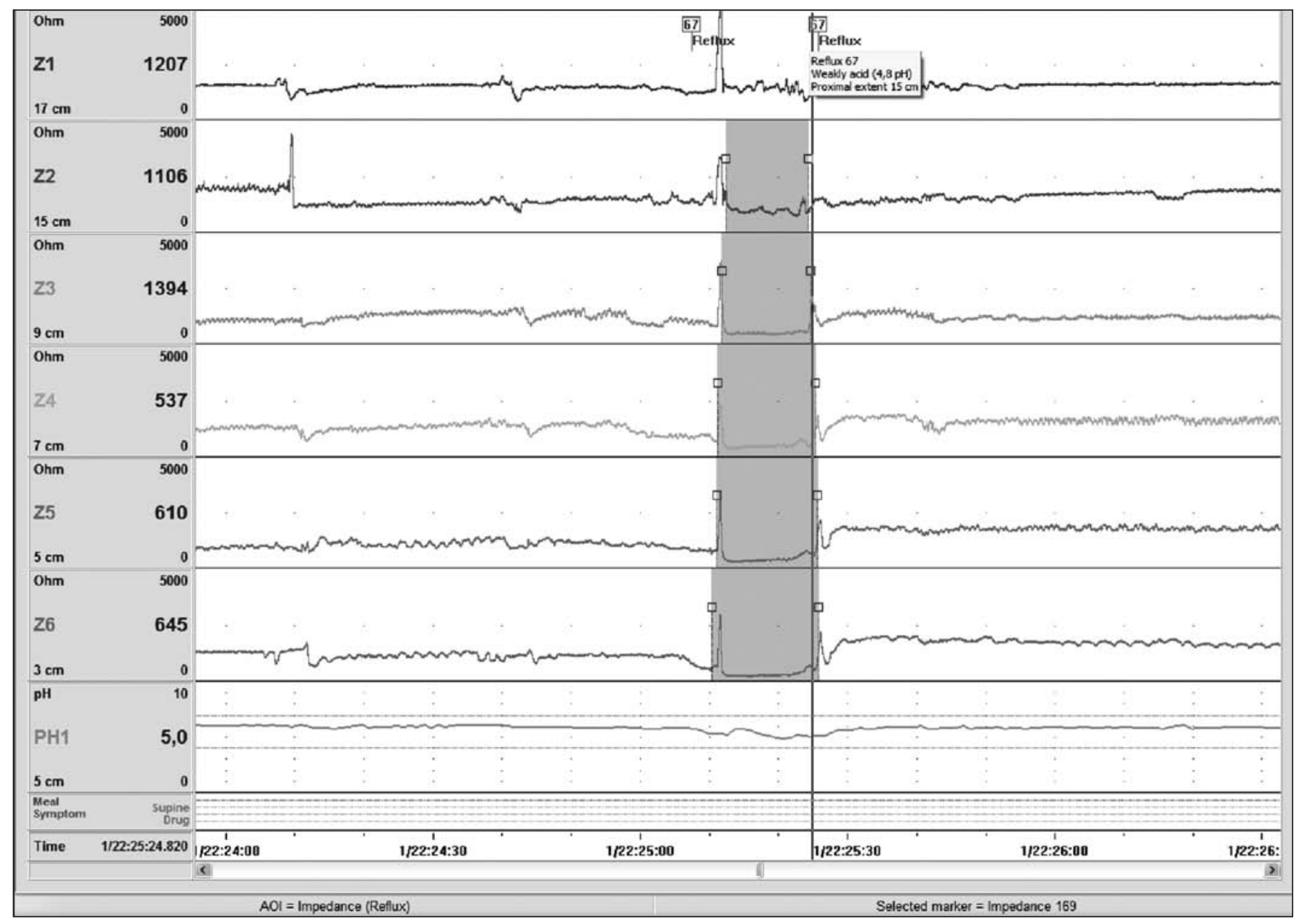

Fig. 1. Imagen de impedancia esofágica multicanal-pH demostrando la presencia de reflujo (véase progresión retrógrada ascendente del material refluido) con progresión proximal incompleta (no alcanza el detector más proximal; Z1). El reflujo es de tipo moderadamente ácido ya que experimenta una caída de pH desde 5,7 a 4,6. Este reflujo no sería detectado en una pHmetría convencional.

con caída rápida denota la entrada del bolo (que se mantendrá mientras esté presente), la IV indicará la contracción esofágica con evacuación del alimento y la $\mathrm{V}$ indica la vuelta al estado basal. Todo ello nos permite analizar hechos tan importantes en motilidad como el conocer el tiempo real de progresión del bolo, la velocidad del mismo, las características y alcance del reflujo y las alteraciones de propulsión segmentarias o generalizadas (4).

Desde los primeros trabajos en el año 1991 de Silny y cols. (5), la IIM ha experimentado una gran progresión en el conocimiento de los patrones de motilidad normales y anormales tanto en el niño como en el adulto. Ello ha permitido ampliar nuestro conocimiento en la patología motora del tubo digestivo, y en especial de la esofágica (6), realizando un complemento perfecto a técnicas como la pHmetría y manometría, caracterizando nuevas entidades como el reflujo moderadamente ácido (medición de reflujo por IIM con descenso de al menos 1 punto en el pH esofágico siempre por encima de 4), reflujo no ácido (existencia de reflujo por IIM sin fluctuación del pH esofágico) y re-reflu- jo (existencia de un nuevo episodio de reflujo cuando todavía el detector de $\mathrm{pH}$ no ha normalizado el registro por encima de 4, este factor hace imposible por el observador la distinción de dos reflujos separados en la pHmetría convencional) (7). Así pues la IIM complementa el estudio del tránsito del bolo a tiempo real correlacionándolo con la motilidad y pH esofágico. La asociación de manometría e IIM permitió a Tutuian y Castell en el año 2004 (8) relacionar las anomalías en la función motora esofágica por manometría con una evaluación de la propulsión correcta o incorrecta del bolo esofágico con IIM en 350 pacientes. Llegaron a la conclusión que entidades como la esclerodermia y la acalasia nunca presentaban una motilidad normal, que otras patologías como el esófago en sacacorchos siempre tenían la motilidad conservada, y que dos entidades tan prevalentes como el espasmo esofágico y la peristalsis esofágica ineficaz (PEI) podían presentar alteraciones en la propulsión del bolo alimentario en al menos la mitad de los enfermos (con una intensidad variable en función de si el material era líquido o viscoso). Esto tiene importantes 
repercusiones porque, en primer lugar, define a la IIM como la prueba de mayor validez a la hora de plantear opciones quirúrgicas en pacientes con PEI y, en segundo lugar, porque permite diferenciar dentro de estas entidades distintos grados de afectación en función de la alteración de la motilidad esofágica (leve si no la hay, moderada si sólo presenta dificultad de propulsión a semisólidos y grave si también lo hace a líquidos).

La información a nivel cualitativo y cuantitativo sobre las características del reflujo que nos aporta la IIM ha revolucionado nuestro conocimiento sobre la enfermedad por reflujo gastroesofágico (ERGE). Podemos considerar hoy en día a la IIM asociada a la pHmetría como el patrón oro en el estudio de la ERGE. En adultos está demostrado por técnicas de $\mathrm{pH}$-IIM que el número de reflujos entre un paciente con sintomatología típica de reflujo y un individuo asintomático es similar, pero que el primero presenta una mayor proporción de reflujos ácidos que el segundo (6). También conocemos que en pacientes con sintomatología refractaria a inhibidores de la bomba de protones (IBP) (9) o con sintomatología atípica (respiratoria, laríngea) sólo en un $20 \%$ de los casos encontraremos un mal control del reflujo ácido, y que hasta en un $40 \%$ de los pacientes apreciaremos reflujo de características no-ácidas, con buena correlación con la clínica (10). Este hecho nos permite cambiar la actitud terapéutica hacia otras opciones como cirugía, sucralfato, baclofeno o la modificación de la pauta actual de consumo de IBP que efectúe el paciente (11), para lo cual y sin la determinación de estudios funcionales como la IIM no se hubieran podido adoptar. Se han propuesto algoritmos diagnósticos para la ERGE donde la pH-IIM ocuparía el lugar actual de la pHmetría aislada (8).

En niños de corta edad el reflujo no-ácido adquiere su vertiente más dramática (12). Esto es así dado que el reflujo que más comúnmente se encuentra en neonatos y lactantes es de tipo postprandial y predominantemente no ácido, denotando la inmadurez funcional del esófago y del esfínter esofágico inferior (13). En el estudio de Wenzl y cols. (14) con pH-IIM se demostró que el reflujo alcanzaba el electrodo a nivel faríngeo en el $71 \%$ de las ocasiones y, lo más importante, hasta en el $86 \%$ de los eventos de reflujo se encontraron alteraciones respiratorias (apnea, desaturación) tanto más importantes como más prolongados eran los episodios de reflujo. Sólo en el $12 \%$ de los casos el reflujo era ácido $(\mathrm{pH}<4)$, lo cual lo haría indetectable para la pHmetría aislada. Es por ello que la IIM sea considerada como técnica de elección en edad neonatal para el estudio de la ERGE (15).

En nuestro caso la paciente presentaba un largo historial de exploraciones enfocadas al diagnóstico y control de una ERGE no erosiva, refractaria al tratamiento correcto con inhibidores de la bomba de protones, cuyo síntoma principal era la tos. La exploración demostró la existencia de un reflujo patológico moderadamente ácido (indetectable por pHmetrías convencionales previas) a pesar del tratamiento con rabeprazol. La exploración por impedancia descartaba que tanto los episodios de reflujo ácido como los no ácidos tuvieran una correlación significativa con la aparición de la sintomatología de la paciente, lo que permitió evitar nuevas y costosas exploraciones enfocadas al diagnóstico de un reflujo patológico inexistente y el empleo permanente de fármacos destinados al control del pH gastroesofágico.

Con todos estos hechos es previsible que en los próximos años la impedancia esofágica multicanal asociada a la pHmetría conforme uno de los métodos de elección en la enfermedad por reflujo gastroesofágico, especialmente en los casos de refractariedad a los tratamientos habituales, una vez definidas de forma clara las implicaciones clínicas que tienen acontecimientos como el reflujo no ácido o moderadamente ácido.

\section{BIBLIOGRAFÍA}

1. Tutuian R, Vela MF, Shay SS, Castell DO. Multichannel intraluminal impedance in esophageal function testing and gastroesophageal reflux monitoring. J Clin Gastroenterol 2003; 37 (3): 206-15.

2. Shay SS, Richter JE. Importance of additional reflux events during esophageal acid clearing. Dig Dis Sci 1998; 43 (1): 95-102.

3. Nguyen HN, Silny J, Matern S. Multiple intraluminal electrical impedancometry for recording of upper gastrointestinal motility: Current results and further implications. Am J Gastroenterol 1999; 94 (2): 306-17.

4. Sifrim D, Holloway R, Silny J, Tack J, Lerut A, Janssens J. Composition of the postprandial refluxate in patients with gastroesophageal reflux disease. Am J Gastroenterol 2001; 96 (3): 647-55.

5. Silny J. Intraluminal multiple electric impedance procedure for measurement of gastrointestinal motility. Neurogastroenterol Motil 1991; 3: $151-62$.

6. Sifrim D, Holloway R, Silny J, Xin Z, Tack J, Lerut A, et al. Acid, nonacid, and gas reflux in patients with gastroesophageal reflux disease during ambulatory 24-hour $\mathrm{pH}$-impedance recordings. Gastroenterol 2001; 120 (7): 1588-98.

7. Shay SS, Johnson LF, Richter JE. Acid reflux: A review, emphasizing detection by impedance, manometry, and scintigraphy, and the impact on acid clearing pathophysiology as well as interpreting the $\mathrm{pH}$ record. Dig Dis Sci 2003; 48 (1): 1-9.

8. Tutuian R, Castell DO. Combined multichannel intraluminal impedance and manometry clarifies esophageal function abnormalities: Study in 350 patients. Am J Gastroenterol 2004; 99 (6): 1011-9.

9. Vela MF, Camacho-Lobato L, Srinivasan R, Tutuian R, Katz PO, Castell DO. Simultaneous intraesophageal impedance and $\mathrm{pH}$ measurement of acid and nonacid gastroesophageal reflux: Effect of omeprazole. Gastroenterol 2001; 120 (7): 1599-606.

10. Tutuian R, Castell DO. Use of multichannel intraluminal impedance to document proximal esophageal and pharyngeal nonacidic reflux episodes. Am J Med 2003; 115 (Supl. 3A): 119S-23S.

11. Vela MF, Tutuian R, Katz PO, Castell DO. Baclofen decreases acid and non-acid post-prandial gastro-oesophageal reflux measured by combined multichannel intraluminal impedance and $\mathrm{pH}$. Aliment Pharmacol Ther 2003; 17 (2): 243-51.

12. Orenstein SR. An overview of reflux-associated disorders in infants: Apnea, laryngospasm, and aspiration. Am J Med 2001; 111 (Supl. 8A): $60 \mathrm{~S}-3 \mathrm{~S}$.

13. Orenstein SR. Infantile reflux: Different from adult reflux. Am J Med 1997; 103 (5A): 114S-9S.

14. Wenzl TG. Evaluation of gastroesophageal reflux events in children using multichannel intraluminal electrical impedance. Am J Med 2003; 115 (Supl. 3A): 161S-5S.

15. López Alonso M, Moya MJ, Cabo JA, Ribas J, Macias MC, Silny J, et al. Acid and non-acid gastro-esophageal reflux in newborns. Preliminar results using intraluminal impedance. Cir Pediatr 2005; 18 (3): 121-6. 